Chirurg 2022 $\cdot 93: 342-348$

https://doi.org/10.1007/s00104-022-01596-w

Angenommen: 18. Januar 2022

Online publiziert: 23. Februar 2022

(c) The Author(s), under exclusive licence to Springer Medizin Verlag GmbH, ein Teil von Springer Nature 2022

Redaktion

U. Settmacher, Jena

\section{Mindestmengenregelungen in der Chirurgie aus Sicht der Fachgesellschaft (DGCH)}

\section{Spagat zwischen Wissenschaft, Politik, Versorgungsrealität und einer Reihe weiterer Aspekte}

\author{
Hauke Lang ${ }^{1}$ Peter Philipp Grimminger ${ }^{1} \cdot$ Hans-Joachim Meyer ${ }^{2}$ \\ 'Klinik für Allgemein-, Viszeral- und Transplantationschirurgie, Universitätsmedizin der Johannes- \\ Gutenberg-Universität Mainz, Mainz, Deutschland \\ ${ }^{2}$ Generalsekretär der Deutschen Gesellschaft für Chirurgie, Berlin, Deutschland
}

\title{
Zusammenfassung
}

\section{In diesem Beitrag}

- Aktueller Anlass der Debatte

- Historie der Mindestmengen in der Onkochirurgie

- Gesetzliche Mindestmengenvorgaben in Deutschland

- Wissenschaftliche Evidenz und Effekte der Mindestmenge am Beispiel der Ösophaguschirurgie

- Kritische Diskussion aus Sicht der Fachgesellschaft

- Auswirkungen der Novellierung der Mindestmenge Ösophagus

- Die bisherige Mindestmengenregelung Ösophagus

- Fahrzeitenverlängerung für die Patienten zu den Kliniken, poststationäre Komplikationsbehandlung

- Noch fehlende Kooperationsverbünde/ -verträge

- Problem der Ressourcenknappheit in den bestehenden Zentren

- Chirurgische Forschung und chirurgische Weiterbildung

- Die Versorgungsrealität und Bedarfsplanung am Beispiel Nordrhein-Westfalen

- Weitere Aspekte

Die wissenschaftlich begründete, fachchirurgische Diskussion um die gesetzliche Vorgabe von Mindesteingriffszahlen für diverse Organsysteme und ausgewählte operative Maßnahmen als Basis einer Qualitätssicherung und Versorgungsoptimierung ist nicht neu. Hierzu liegen umfangreiche und auch belastbare Daten aus nationalen und internationalen Studien für die kolorektale Chirurgie, die Pankreaschirurgie, die Ösophaguschirurgie, die Leberchirurgie und die Magenchirurgie vor. Jüngst hat die Anhebung der Mindestmenge für komplexe Eingriffe am Ösophagus durch den Gemeinsamen Bundesausschuss (G-BA) von 10 auf 26 pro Klinik und Jahr die Debatte um dieses Thema, aber auch die Debatte über die Zentralisierung im Gesundheitswesen im Generellen neu aufgerollt. Die Anhebung erscheint aus Sicht der politischen Gremien wissenschaftlich gut begründet und in der praktischen Umsetzung realisierbar. Aus Sicht der tagtäglich mit den entsprechenden hochkomplexen Entitäten vertrauten Ärzten ergibt sich allerdings eine sehr viel breitere Diskussionsgrundlage, welche nur partiell durch ein Gutachten des Instituts für Qualität und Wirtschaftlichkeit im Gesundheitswesen (IQWiG) als Grundlage des G-BA-Beschlusses abgedeckt wird. Für die wissenschaftlich orientierte chirurgische Fachgesellschaft steht dabei in erster Linie die wissenschaftliche Evidenz als Handlungsmaxime im Vordergrund. Gleichwohl können und dürfen Aspekte der Versorgungsrealität nicht ausgeblendet werden. Die Empfehlungen der Fachgesellschaft müssen sich somit neben der Ergebnisqualität auch an der praktischen Realisierbarkeit orientieren. Darüber hinaus sind Fragen der Weiterbildung, das Recht des Patienten auf freie Arztwahl sowie auch der Erhalt der Attraktivität des Berufsbildes Chirurg immanente Themen der chirurgischen Fachgesellschaft.

\section{Schlüsselwörter}

Qualitätssicherung · Versorgungsoptimierung · Versorgungsrealität · Evidenz · Weiterbildung

\section{Hintergrund}

Es gilt als grundsätzlich belegt, dass die Ergebnisqualität operativer Maßnahmen positiv korreliert mit der Fallzahl dieser Eingriffe [1-3]. Dabei kann sich die Ergebnisqualität in sehr globalen, harten Endpunkten wie der Klinikletalität, der Morbidität (z. B. nach Clavien-Dindo) oder auch in sehr distinkten Parametern wie Opera- tions- und Krankenhausverweildauer, Zeit der Einlungenventilation, Reinterventionsrate, Bluttransfusionsbedarf, Konversionsrate etc. ausdrücken $[2,3,7]$. Bei der Fallzahl können wiederum die Gesamteingriffszahl pro Operateur („Lernkurve“), die Eingriffszahl pro Operateur und Jahr ("Eingriffsroutine") oder die Fallzahl pro "chirurgischer Einheit" einer Klinik zugrunde gelegt werden. Aus abhängigen Variablen und 
Schwellenwerten können so Mindestfallzahlen generiert werden, in der Absicht, die Ergebnisqualität zu steigern oder auf einem hohen Niveau zu garantieren $[2,7]$.

Dieser Prozess wirkt transparent und logisch. Allerdings ist bei eingehender Betrachtung und entsprechender klinischer Erfahrung im Umgang mit den jeweiligen Krankheitsbildern und den Operationen zu erkennen, dass die konkrete Festlegung von Fallzahlen durchaus mit Schwierigkeiten verbunden ist. So sind die individuellen Lernkurven pro Eingriff sehr variabel, auch die erforderliche Eingriffsroutine ist individuell verschieden. Die strukturellen Gegebenheiten, die 24-Stunden/7-Tage(24/7)-Verfügbarkeit und die fachliche Leistungsfähigkeit von Nachbardisziplinen - wie etwa einer interventionellen Radiologie und Endoskopie und einer Hochleistungsintensivmedizin - sind von Klinikstandort zu Klinikstandort unterschiedlich. Auch die Patientenkollektive unterscheiden sich hinsichtlich ihres Komplikationspotenziales von Klinik zu Klinik. Zudem wirken sich Zentrumseffekte wie z. B. Patientenselektion oder die stärkere Einbindung von Patienten in klinische Studien auf die Morbidität und Letalität aus, ohne dass dies primär im Zusammenhang mit der rein operativen Expertise und Ergebnisqualität steht. Umgekehrt werden in größeren Zentren erwiesenermaßen aber auch vermehrt Fälle mit erhöhter Fallschwere behandelt, was sich wiederum negativ auf Morbidität und Letalität auswirken kann.

Die Interpretation der verfügbaren wissenschaftlichen Daten zu diesen Themenkomplexen sind daher entsprechend anspruchsvoll. Die wirklich belastbare Datenlage für die Formulierung sehr konkreter Fallzahlen im Sinne von Mindestmengen ist daher überschaubar und sehr eingeschränkt $[3,4,7]$.

\section{Aktueller Anlass der Debatte}

Zum 01.01.2021 erfolgte durch Verordnung des Gemeinsamen Bundesausschuss (G-BA) in Deutschland die Anpassung der Mindestmengenregelung (MM-R) für resezierende komplexe Eingriffe am Organsystem Ösophagus für Erwachsene von bisher 10 auf jetzt 26 Eingriffe je Standort und Jahr (\$136b Absatz 1 Nummer 2 Sozialgesetzbuch [SGB] V für nach $\$ 108$ zuge- lassene Krankenhäuser nach Anlage Nr. 3; [5]). Aus wissenschaftlicher und fachchirurgischer Sicht besteht dabei grundsätzlich Einigkeit, dass die hiermit verbundene Zentralisierung von Speiseröhrenoperationen die Chance auf eine weitere Verbesserung der Versorgungsqualität in sich birgt $[6,7]$. Die konkrete Zahl von 26 ist dabei jedoch umstritten und diskutabel. Neben der „nackten“ Zahl der Eingriffe muss eine Vielzahl weiterer Aspekte Berücksichtigung finden. Zudem bedarf die klinische Umsetzung der Mindestmengenregelung deutlich mehr Vorbereitungszeit als der Gesetzgeber dies vorgibt [7].

Weitere Veränderungen wurden für die Zeit ab 2025 vom G-BA am 16.12.2021 bereits beschlossen. So wurde die Zahl für resezierende Eingriffe am Pankreas von 10 auf 20 erhöht. Für das Lungenkarzinom wurde eine Mindesteingriffszahl von 75 und für das Mammakarzinom von 100 pro Jahr und Klinikfestgelegt. Auch für das tiefe Rektumkarzinom, für das Magenkarzinom und für kinderchirurgische Eingriffe sind Vorgaben und weitere Anpassungen zu erwarten.

\section{Historie der Mindestmengen in der Onkochirurgie}

Bereits vor 20 Jahren verwiesen Publikationen auf eine signifikant verbesserte Ergebnisqualität - v.a. bezüglich des Endpunktes Letalität - bei Tumoroperationen (Lunge, Ösophagus, Magen, Rektum, Pankreas, Leber) an sog. "High-volume-Zentren“ im Vergleich zu Kliniken mit geringerer Operationsfrequenz. Seit 1957 werden entsprechende Kollektive ausgewertet $[1,3,8]$. Auch wurde belegt, dass die Parameter Behandlungskosten und Krankenhausverweildauer bei hohen Fallzahlen signifikant gesenkt werden können.

\section{॥ Das verbesserte Outcome an High-volume-Kliniken ist eng verknüpft mit strukturellen Voraussetzungen}

Dabei bezogen sich die Ergebnisse bereits früh nicht nur auf die Eingriffshäufigkeit pro Operateur, sondern vielmehr auf die Eingriffshäufigkeit pro chirurgische Einheit. Das verbesserte Outcome an Highvolume-Kliniken ist somit eng verknüpft mit den strukturellen Voraussetzungen hinsichtlich des in der Regel interdisziplinären Komplikationsmanagements. Neben der individuellen Expertise, in erster Linie beruhend auf operativem Geschick sowie Erfahrung des Chirurgen, z.B. bei der Indikationsstellung und beim Komplikationsmanagement, sind für eine entsprechende Ergebnisqualität auch leistungsfähige und 24/7 verfügbare Partner in den Nachbardisziplinen wie interventionelle Endoskopie und Radiologie, Intensivmedizin oder Kardiologie notwendig (Prozess- und Strukturqualität).

In der Pankreaschirurgie wurde von Krautz und Mitarbeitern, welche über 60.000 Pankreaseingriffe in Deutschland auswerteten, eine Klinikletalität von 6,5\% in sog. "Very-high-volume-Kliniken" (median 105 Eingriffe/Jahr) vs. 11,5\% in „Verylow-volume-Kliniken" (median 4 Eingriffe/ Jahr) nachgewiesen. Die Interventionsrate wegen Komplikationen nach Pankreaseingriffen betrug $24,2 \%$ in den Very-highvolume-Kliniken und $36,8 \%$ in den Verylow-volume-Kliniken [9].

In der kolorektalen Chirurgie wurde in Deutschland von der Würzburger Arbeitsgruppe um Germer eine um ein Drittel reduzierte Letalität konstatiert, wenn die Eingriffe in nach Deutscher Krebsgesellschaft zertifizierten Zentren durchgeführt werden [10]. Untersuchungen bei der transanalen Rektumchirurgie (transanale totale mesorektale Exzision, TaTME) zufolge war die Konversionsrate in High-volume-Kliniken (über 30 TaTME/Jahr) fast halbiert im Vergleich zu Nicht-High-volume-Kliniken (unter $30 \mathrm{TaTME} / J a h r$ ) bei zudem signifikant besserer Qualität der TME-Präparate (zirkumferenzieller Resektionsrand; [11]).

Umfangreiche allgemeinchirurgische Literatur steht auch für Eingriffe bei chronisch-entzündlichen Darmerkrankungen, für die Schilddrüsenchirurgie, die bariatrische Chirurgie und die Chirurgie der komplexen Hernien zur Verfügung. Auch diese Daten belegen, dass Eingriffe an Zentren mit hoher Fallzahl sicherer sind und zu einer besseren Ergebnisqualität führen.

Meyer verwies bereits 2005 in seiner Arbeit im European Journal of Surgical Oncology zum Magenkarzinom auf die klare Evidenzlage bezüglich des „Klinikvolumens" - weniger der individuellen ope- 
rativen Expertise des Operateurs - in der Magenkarzinomchirurgie hinsichtlich der Ergebnisqualität. Hier wurde jedoch auch betont, dass die Festlegung auf exakte Schwellenwerte mit erheblichen Schwierigkeiten verbunden ist [12].

\section{Gesetzliche Mindestmengen- vorgaben in Deutschland}

Die verfügbare wissenschaftliche Evidenz wurde seitens des Gesetzgebers bisher nur bei wenigen definierten Eingriffe umgesetzt. Daher wurde die Mindestmengenregelung nicht nur als "never ending story", sondern auch als "zahnloser Papiertiger" - im Sinne des Fehlens versorgungs- und vergütungsrelevanter Konsequenzen durch die Kostenträger bei Nichtbeachtung - bezeichnet. Aufgrund von "Kulanz" oder Ausnahmetatbeständen wie etwa fachliche Neuausrichtung oder Leitungswechsel der chirurgischen Einheit vergüteten die Krankenkassen bislang häufig auch beim Nichterreichen der Kennzahlen. Ein Zentralisierungseffekt oder zumindest relevanter Einfluss auf die Versorgungsstrukturen blieb daher bislang aus [13].

Die Mindestmengenregelungen des G-BA umfasst bisher nur sieben planbare Leistungen:

- Lebertransplantation (inklusive Teilleberlebendspende),

- Nierentransplantation (inklusive Lebendspende),

- komplexe Eingriffe am Organsystem Ösophagus,

- komplexe Eingriffe am Organsystem Pankreas,

- Stammzelltransplantation,

- Kniegelenktotalendoprothesen,

- Versorgung von Früh- und Neugeborenen mit einem Geburtsgewicht von unter $1250 \mathrm{~g}$.

Wie eingangs erwähnt, kommen ab 2025 Mindestmengen für das Mammakarzinom und das Lungenkarzinom hinzu.

Nimptsch et al. (2016) verglichen für diese Prozeduren die Letalität in Kliniken, welche die Mindestmengenvorgaben einhielten, gegenüber solchen, welche diese Vorgaben nicht erfüllten. Dies ergab für die Ösophaguschirurgie eine Letalität von $9,2 \%$ vs. $12,1 \%$, für die Pankreaschirur- gie von $8,6 \%$ vs. $11,8 \%$. Bei den Nierentransplantationen ergab sich eine Letalität von 1,7\% in Kliniken oberhalb der Mindestmengenvorgaben vs. 3,3\% in Kliniken unterhalb der Mindestmengenvorgaben, für Kniegelenkersatzoperationen waren die endsprechenden Ergebnisse mit $0,13 \%$ vs. $0,18 \%$ fast identisch. Bei den Lebertransplantationen (15,9 vs. $15,5 \%$ ) und Stammzelltransplantationen (6vs.4\%) war die Letalität an den High-volume-Kliniken sogar etwas höher als an den Low-volume-Kliniken, was jedoch mit einer erhöhten Fallschwere interpretiert werden muss [14].

\section{Wissenschaftliche Evidenz und Effekte der Mindestmenge am Beispiel der Ösophaguschirurgie}

Operationen an der Speiseröhre sind hochspezialisierte, komplexe, aber auch seltene Eingriffe. Sie sind in der Regel planbar und daher grundsätzlich mindestmengenfähig. Es handelt es sich bei der überwiegenden Mehrzahl um Eingriffe wegen eines Karzinoms, so im Jahr 2015 in $84 \%$ (3697/4032 Eingriffe) bundesweit [2]. In mehreren Arbeiten wurde konstatiert, dass ein erfahrener Ösophaguschirurg mit hoher individueller Operationsfrequenz und signifikanter Überschreitung der „Lernkurve" eine um bis zu $50 \%$ niedrigere Klinikletalität und - in geringerem Maße auch verbessertes Langzeitüberleben als ein Operateur mit geringerer Erfahrung erreichen kann.

\section{》) Ergebnisqualität hängt nicht allein von der operativen Leistung $a b$}

Ebenso wurde bei erfahrenen Ösophaguschirurgen auch eine signifikant geringere Komplikationsrate nachgewiesen. Mit steigender Routine des Ösophaguschirurgen sinkt häufig auch die Operationszeit und somit die Dauer der Einlungenventilation bei gleichzeitig auch niedrigerem Blutverlust und -transfusionsbedarf sowie kürzerer Verweildauer auf der Intensivstation und in der Klinik insgesamt. Gleichzeitig steigt mit der Routine des Ösophaguschirurgen auch die "Lymphknotenausbeute" als ein Parameter für die onkologische Qualität der Operation. Die Ergebnisse wurden gleichermaßen in Studien aus den USA, England, Holland und Dänemark bestätigt.

Betrachtet man die Anzahl der Operationen pro Abteilung und nicht die individuelle Operationsfrequenz des Operateurs, so konnte ebenfalls eine signifikante Abnahme der Letalität mit steigender Anzahl von Ösophagusresektionen pro Jahr gezeigt werden $[4,15,16]$. Nimptsch et al. analysierten 22.700 Behandlungsfälle von 2010 bis 2015 in Deutschland. Es fand sich eine signifikant geringere Letalität $(6,8 \%)$ in Kliniken mit median 62 Fällen pro Jahr als in Kliniken mit nur 2 Fällen pro Jahr $(12,2 \%)$ bei jedoch festgestellt gleicher Komplikationshäufigkeit von über $50 \%$. Dies ist ein eindeutiger Hinweis, dass Ergebnisqualität nicht allein von der operativen Leistung abhängt, sondern vielmehr von dem durch Prozess- und Strukturqualität geprägten Komplikationsmanagement („ffailure to rescue ${ }^{\prime \prime}$ ). Die Autoren betrachteten die jährliche Klinikleistungsmenge als Variable und ermittelten einen erforderlichen Schwellenwert von 26 Operationen pro Jahr, bei dem die im Beobachtungszeitraum bundesweit durchschnittliche Letalität von 9,5\% bei Ösophagektomien unterschritten wird. Diese Betrachtung ist die Basis der Beschlussfassung des G-BA [2].

\section{Kritische Diskussion aus Sicht der Fachgesellschaft}

Aufgabe und Selbstverständnis der Fachgesellschaft ist neben der Verbreitung wissenschaftlich gesicherter Kenntnisse (Empfehlungen oder Leitlinien) sowie der Aus-, Fort- und Weiterbildung der Mitglieder auch die Vertretung standespolitischer Interessen. Die Fachgesellschaften müssen alle diese Aspekte in Beratungen mit politischen Entscheidungsträgern adressieren, z.B. bei der Diskussion um strukturelle Rahmenbedingungen bzw. Veränderungen solcher. Zu sämtlichen Einführungen und Novellierungen der Mindestmengenregelungen seitens des Gesetzgebers haben die assoziierten Fachgesellschaften auch stets versucht, frühzeitig Stellung zu nehmen. Naturgemäß können dabei im Spagat zwischen Sachkompetenz und politischen Interessenslagen Konfliktsituationen entstehen, zumal die Gesetzgeberseite die Empfehlungen der 
Fachgesellschaften in der Regel nicht vollumfänglich übernimmt.

\section{Auswirkungen der Novellierung der Mindestmenge Ösophagus}

Die mutmaßlichen Auswirkungen der Mindestmengenregelung auf die Kliniklandschaft in Deutschland und die Versorgungsrealität sind gravierend und in diesem Kontext viele Fragen noch ungeklärt. Allein die Tatsache, dass überhaupt nur knapp über 20 Kliniken in Deutschland im Jahr 2015 mehr als 25 Ösophaguseingriffe durchführten, lässt die geplante Anpassung in einem neuen Licht erscheinen. Es bedeutet konsequenterweise das Aus für planbare Ösophagusoperationen für die knapp weiteren 400 Kliniken in Deutschland, welche bislang niederfrequent Operationen an der Speiseröhre durchführten.

Die Umsetzung kommt plötzlich und trifft die Kliniken, Klinikträger- und die Versorgungsstrukturen weitestgehend unvorbereitet. Die „Vorbereitungszeit" in den Jahren 2021 und 2022, die Zeit der sog. Übergangsregelung mit weiterhin erlaubten 10 Eingriffen pro Standort bis zur definitiven "Scharfschaltung" im Jahr 2023, ist knapp. Auch waren zur Zeit des Gesetzesentwurfs die Parallelentwicklungen einer weltweiten COVID-19 ("coronavirus disease 2019")-Pandemie und die massive Zunahme des Intensivpflege- und Operationsfachkräftemangels nicht vollständig vorhersehbar.

An diversen Schlüsselpositionen ist jetzt Engagement gefragt, um die unbestritten in vielerlei Hinsicht sinnvolle Anpassung des G-BA zu einer Qualitätsverbesserung mit Vorteilen für die Patienten werden zu lassen und dies, ohne gleichzeitig nachteilig für die Gesundheitsdienstleister zu werden. Darüber hinaus müssen aber auch andere Aspekte wie etwa die Weiterbildung und die Attraktivität des Berufsbildes "Chirurg" unbedingt Berücksichtigung finden.

\section{Die bisherige Mindestmengen- regelung Ösophagus}

Die am 21.06.2006 auf 10 Eingriffe pro Jahr festgelegte Mindestmenge wurde von der überwiegenden Mehrzahl der Kliniken,
welcheÖsophagusoperationen durchführten, nicht eingehalten. In den Kliniken in Deutschland, die überhaupt Eingriffe an der Speiseröhre durchführen, wurden im Median lediglich 7 Eingriffe durchgeführt [2].

\section{Fahrzeitenverlängerung für die Patienten zu den Kliniken, poststationäre Komplikationsbehandlung}

Zur Erörterung und Bewertung des vom G-BA selbst formulierten Problems der Fahrzeiten wurde das IQTiG (Institut für Qualitätssicherung und Transparenz im Gesundheitswesen) mit einem Gutachten beauftragt. Patienten hatten bislang zu der Klinik, in der sie eine Speiseröhrenoperation vornehmen ließen, im Schnitt 20 min Fahrzeit und eine Wegstrecke von $17 \mathrm{~km}$ zurückzulegen. Bei der zu erwartenden Zentralisierung durch Anhebung der Mindestmenge auf 26 pro Klinik und Jahr würde sich die mittlere Fahrzeit auf $31 \mathrm{~min}$ (plus $11 \mathrm{~min}$ ) und die Wegstrecke auf $36 \mathrm{~km}$ (plus $19 \mathrm{~km}$ ) verlängern. Dies wurde mit Umverteilungsalgorithmen und Modellberechnungen ermittelt [2].

Für die operative Primärbehandlung müsste somit bei Umsetzung der Mindestmengen im Median eine nur geringfügig längere Wegstrecke in Kauf genommen werden, was bei der elektiven Form der Operation trotz sicherlich vorhandener Unwägbarkeiten im Einzelfall ohne Zweifel akzeptabel ist. Allenfalls könnten durch die längere Wegstrecke im Falle poststationärer Komplikationen, wie Dysphagie, Verschlechterung des Allgemeinzustandes oder Ernährungsprobleme sowie insbesondere bei der sehr seltenen, aber u.U. perakuten und vital bedrohlichen Komplikation eines - eingriffstypischen Enterothorax nach Ösophagektomie und Magenhochzug, ,logistische Schwierigkeiten" bis zum Erreichen einer entsprechend spezialisierten Klinik auftreten, was aber durch die dann vor Ort gesichert vorhandene Expertise mehr als kompensiert sein dürfte [17].

\section{Noch fehlende Kooperations- verbünde/-verträge}

Erstdiagnostik, leitlinienkonformes Primärstaging, prätherapeutische Vorstellung im Ösophaguszentrum mit Risikostratifizierung, Tumorboardvorstellung mit entsprechender Zuteilung zu den multimodalen Therapiepfaden und auch Prozesse wie die Prähabilitation können in der Gesamtheit nur durch leistungsstarke und in der Zusammenarbeit erprobte und bewährte Kooperationen erfolgen. Denkbar sind Verbünde aus kleineren Kliniken mit jedoch leistungsfähigen gastroenterologischen und radiologischen Abteilungen und den bestehenden bzw. auch den avisierten zukünftigen Zentren der Ösophagusmedizin. Ohne solche Verbünde entstünde ein immenser und wohl kaum zu bewältigender Mehraufwand für die wenigen verbleibenden Zentren.

\section{》) Stabile Zuweisungs- und Kooperationsstrukturen bestehen nur in wenigen Kliniken}

Die Notwendigkeit eines Kooperationsnetzwerkes ergibt sich auch aus den bereits angesprochenen Aspekten des Komplikationsmanagements (Stenose, Spätinsuffizienz, Enterothorax) und der Tumornachsorge. Es liegt in der Komplexität der Ösophaguserkrankungen begründet, dass Patienten häufig und aufgrund der mittlerweile deutlich verbesserten Therapiemöglichkeiten erfreulicherweise auch viele Jahre postoperativ noch von Gastroenterologen, Onkologen und eben auch dem Operateur betreut werden müssen. Durch vermehrte "Salvage-Konzepte“, aber auch weiter steigende Überlebensraten durch die Erfolge der multimodalen Therapie wird diesem Umstand in Zukunft noch weitere Bedeutung zukommen. Stabile Zuweisungs- und Kooperationsstrukturen und ein professionelles Zweitmeinungsmanagement bestehen aktuell nur in wenigen Kliniken, vorzugsweise in den wenigen High-volume- und Exzellenzzentren für die Chirurgie der Speiseröhre und des oberen Gastrointestinaltraktes.

Dass bis dato kaum Kooperationsverbünde bestehen, dürfte zu einem erheblichen Anteil auch in der bisherigen Wettbewerbssituation der Kliniken, welche der 
Ausbildung solcher Kooperationen eher abträglich war, begründet liegen.

Die in der Begründung des G-BA-Beschlusses formulierte Feststellung, dass flächendeckend Nachsorge- und Kooperationsverbünde bereits bestünden, ist eine nicht hinlänglich belegte Behauptung und trifft - die gesamte Versorgungslandschaft überblickend - sicherlich nicht zu.

\section{Problem der Ressourcenknappheit in den bestehenden Zentren}

Seit Beginn der COVID-19-Pandemie vor mehr als 2 Jahren waren es nicht zuletzt die High-volume-Kliniken, welche in besonderem Maße unter Ressourcenknappheit (Operationskapazität, Intensivbettenkapazität) zu leiden hatten und noch haben. Dies liegt nicht allein in der Ausschöpfung der Intensivkapazitäten durch die COVID19-Pandemie begründet. Parallel wiegt der Fachkräftemangel in Intensiv-, Anästhesieund Operationspflege mindestens genauso schwer. Die Gründe des Personalmangels sind vielschichtig. Sie liegen u. a. auch in Fehlern der Berufspolitik der vergangenen Dekade, fehlenden Möglichkeiten der Vereinbarkeit von Familie und Beruf, Abwanderung in alternative Berufsfelder, anhaltender und zunehmender Leistungsdruck in Kliniken, aber auch in Überlastung und Erschöpfung der Fachkräfte - mit oder ohne Einsatz auf COVID-Stationen. Klar ist, dass dies nicht nur ein kurz-, sondern vor allem ein mittel- und langfristiges Problem darstellt. Auch im ärztlichen Sektor ist der Fachkräftemangel in der Viszeralchirurgie seit Jahren spürbar.

Nur 12 Kliniken in Deutschland behandelten im Jahr 2015 mehr als 62 Ösophaguskarzinome mittels radikaler Resektion und nur 26 Kliniken führten über 26 Ösophagektomien durch. Demgegenüber haben im gleichen Jahr 406 Kliniken mindestens eine Ösophagusresektion durchgeführt [2]. Verteilt man 4000 Ösophaguseingriffe auf 69 Kliniken in Deutschland - dies entspricht der Prognose des IQTiG bei Anhebung der Mindestmenge auf 26 pro Klinik und Jahr - so errechnen sich im Durchschnitt etwa 60 Eingriffe pro Jahr und Klinik. Über 50 Ösophagektomien pro Jahr bedeuten jedoch einen hohen personellen und strukturellen Aufwand, der keineswegs "on top“ zu leisten ist, sondern eine signifikante Leistungsausdehnung der jeweiligen Klinik bedeutet. Die geplante Umstrukturierung wäre für viele der dann noch Ösophagusresektionen ausführenden Kliniken unter den gegebenen Rahmenbedingungen kapazitiv und personell anspruchsvoll und nicht ohne Weiteres zu realisieren. Aktuell (Stand 12/2020) gibt es in Deutschland nur 5 Kliniken, Köln (202), Nürnberg (109), Mainz (97), Hamburg (84), und Heidelberg (82), die mehr als 80 Ösophagektomien pro Jahr durchführen [18].

\section{Chirurgische Forschung und chirurgische Weiterbildung}

Universitäre High-volume-Zentren können Weiterbildung und Forschung effektiv gestalten. Innovationen und Fortschritt in der Chirurgie entstammen jedoch keineswegs nur aus den universitären Einheiten. Insbesondere bei der Implementierung und Weiterentwicklung der minimal-invasiven Chirurgie waren und sind nichtuniversitäre Einrichtungen vielfach sogar federführend. Die Pluralität der Behandlung und die extrauniversitäre Forschung war immer ein Motor des medizinischen Fortschrittes, bei dem sich universitäre - und extrauniversitäre Kliniken ergänzten [19].

\section{I) Die Weiterbildungskurrikula sind mit der geplanten Zentralisierung nicht abgestimmt}

Die Umsetzung der Mindestmengenregelung wird weniger mit Einschränkungen in der Forschung als vielmehr auf dem Sektor der Aus- und Weiterbildung einhergehen. Ein gravierendes Problem besteht darin, dass die Weiterbildungskurrikula der speziellen Viszeralchirurgie der Landesärztekammern mit der geplanten Zentralisierung in der Ösophaguschirurgie bisher nicht abgestimmt sind. Hier müssen Rotations- oder Austauschkonzepte zwischen den rein zuweisenden Kliniken und den die Ösophaguschirurgie ausführenden Zentren erarbeitet werden.

\section{Die Versorgungsrealität und Bedarfsplanung am Beispiel Nordrhein-Westfalen}

Derzeit erscheinen die Erfordernisse der Mindestmengenvorgaben noch entkoppelt von der bundesweiten Krankenhausstrukturplanung.

Übergeordnetes Ziel der neuen Krankenhausplanung ist es, Krankenhäusern konkrete medizinische Leistungsbereiche und Leistungen (Operationen, Prozeduren) verbindlich zuzuordnen und damit gekoppelt auch Qualitätskriterien zu definieren. Anfang 2023 sollen Feststellungsbescheide an die Kliniken versandt werden, welche exakt vorgeben, welche Leistungen eine Klinik zukünftig erbringen darf [20].

Erklärtes Ziel ist die Etablierung eines neuen Konzeptes weg von einer reinen Bettenzahlplanung hin zu einer Strukturund Leistungsplanung, die auch an der ärztlichen Weiterbildungsordnung ausgerichtet sein soll. Weiterbildungsverbünde zwischen den Standorten der Spezialversorgung und solchen der Regelversorgung sollen partnerschaftlich gestaltet werden („Kooperation statt Konkurrenz") zum Wohle der Patientenversorgung und der Weiterbildung.

Letztlich entscheidend für den Erfolg der Umsetzung des G-BA-Beschlusses wird auch die Freigabe finanzieller Mittel auf Landes-, Bundes- und Kostenträgerebene sein, um die zwangsläufig zunächst einmal mit Kosten verbundene Zentralisierung finanzieren zu können.

\section{Weitere Aspekte}

Mehrere Kliniken und Klinikverbände bereiten derzeit eine Klage gegen die Umsetzung des G-BA-Beschlusses vor. Kern der Klage ist das Recht der freien Berufsausübung. Somit werden neben den zuvor adressierten Aspekten auch ganz grundsätzliche berufspolitische und ethische Gesichtspunkte aufgegriffen. Dies verdeutlicht die Komplexität der Diskussion. Kritiker der Mindestmengenregelungen geben an, dass Patienten ein Recht auf freie Arztwahl haben. Das Recht des Patienten auf eine individuelle persönliche Behandlung muss zumindest diskutiert, wenn nicht sogar als Einzelfallentscheidung zugelassen werden. 
Schließlich muss die Konzentrierung der medizinischen Versorgung in großen, u. U. heimatfernen Zentren auch vor dem Hintergrund einer immer älter werdenden Bevölkerung mit allen Aspekten (Mobilität, Demenz) und damit verbundenen möglichen Problemen (individuelle Kosten, Zurechtfinden in heimatfernen und großen Strukturen, Angehörigenbesuche) gesehen werden.

Auf diese und andere Fragen müssten aber längst Antworten gefunden sein, sie sind aber zum jetzigen Zeitpunkt in der breiten Diskussion um die Mindestmengendebatten und Zentralisierung in der Medizin zum großen Teil noch nicht einmal angedacht, geschweige denn formuliert.

\section{Fazit für die Praxis}

- Es ist unbestritten, dass sich bei hochkomplexen Prozeduren die Eingriffszahl und das Klinikvolumen günstig auf die Ergebnisqualität auswirken. Demgegenüber ist für exakte Schwellenwerte nach wie vor keine gute Evidenz vorhanden. In der aktuellen Diskussion um Mindestmengen müssen sich die Fachgesellschaften primär an der wissenschaftlichen Evidenz orientieren, in ihren Empfehlungen aber auch die Versorgungsrealität und weitere Aspekte einbeziehen. Dabei dürfen weder die Fachgesellschaften ausschließlich zum verlängerten Arm der Politik noch die Versorgungsökonomie die alleinige Maxime des chirurgischen Handelns werden.

- Es ist jetzt die Initiative aller Kliniken gefragt, Netzwerkstrukturen zu bilden, welche in der Summe eine leitliniengerechte und flächendeckende Versorgung gewährleisten können. Die bestehenden und die Zentren in spe müssen sich personell und strukturell auf ein deutlich höheres Patientenaufkommen vorbereiten.

- Alle weiteren genannten Aspekte einer Mindestmengen- und Zentralisierungsdebatte müssen jetzt aktiv diskutiert und bearbeitet werden, um die wissenschaftlich evidente Datenlage für die Patienten vorteilhaft in der Versorgungsrealität umzusetzen, ohne gleichzeitig die Aus- und Weiterbildung zu gefährden.

Minimum case volume regulations in surgery from the perspective of the specialist society (DGCH). Balancing act between science, politics, treatment reality and a range of other aspects

The scientifically founded surgical specialist discussion regarding the legal requirements for minimum volume numbers for diverse organ systems and selected surgical procedures as the basis of quality assurance and optimization of treatment is not new. Comprehensive and also reliable data from national and international studies are available for colorectal surgery, pancreatic surgery, esophageal surgery, liver surgery and gastric surgery. Recently, the raising of the minimum volume for complex esophageal interventions by the Federal Joint Committee (G-BA) in Germany from 10 up to 26 procedures per hospital and year, reignited the debate on this topic as well as the debate on centralization in the healthcare system in general. This decision seems to be scientifically well-justified from the perspective of political bodies and realizable in the practical implementation; however, from the perspective of physicians routinely involved in the corresponding highly complex procedures, there is a very much broader basis for discussion, which is only partially covered by a report of the Institute for Quality and Efficiency in the Healthcare System (IQWiG) as the foundation of the decision of the G-BA. For the scientifically oriented surgical specialist society, in the first instance priority is given to the scientific evidence as the guiding principle. Nevertheless, aspects of the treatment reality cannot and should not be ignored. Therefore, the recommendations of the specialist society must be oriented not only to the quality of results but also to the realistic options for successful implementation in practice. Furthermore, questions of further education, the right of the patient to freedom of choice of the physician and preservation of the attractiveness of the occupational profile of surgeons are immanent topics for the surgical specialist society.

\section{Keywords}

Quality assurance · Treatment optimization · Treatment reality · Evidence · Advanced education

\section{Korrespondenzadresse}

\section{Prof. Dr. med. Hauke Lang, MA, FACS}

Klinik für Allgemein-, Viszeral- und

Transplantationschirurgie, Universitätsmedizin der Johannes-Gutenberg-Universität Mainz Langenbeckstraße 1, 55131 Mainz, Deutschland

Hauke.Lang@unimedizin-mainz.de

\section{Einhaltung ethischer Richtlinien}

Interessenkonflikt. H. Lang, P.P. Grimminger und H.-J. Meyer geben an, dass kein Interessenkonflikt besteht.

Für diesen Beitrag wurden von den Autoren keine Studien an Menschen oder Tieren durchgeführt. Für die aufgeführten Studien gelten die jeweils dort angegebenen ethischen Richtlinien.

\section{Literatur}

1. Tol JAMG, van Gulik TM, Busch ORC, Gouma DJ (2012) Centralization of highly complex lowvolume procedures in upper gastrointestinal surgery. A summery of systematic reviews and meta-analyses. Dig Surg 29:374-383
2. Nimptsch U, Haist T, Krautz C et al (2018) Fallzahl, Krankenhaussterblichkeit und Komplikationsmanagement in der Ösophaguschirurgie. Analyse deutschlandweiter Krankenhausabrechnungsdaten. Dtsch Arztebl Int 115:793-800. https://doi. org/10.3238/arztebl.2018.0793

3. Begg CB, Cramer LD, Hoskins WJ, Brennan MF (1998) Impact of hospital volume on operative mortality for major cancer surgery. JAMA 280:1747-1751

4. Hölscher AH (2001) Ösophaguskarzinom Operative Therapie in Zentren. Dtsch Ärztebl 98(28-29):A 1890-1894

5. Beschluss des Gemeinsamen Bundesausschusses über eine Änderung der Mindestmengenregelungen: Änderung von $\S 10$ Abs. 1. BAnz AT 28. Juli 2021 B5

6. Hölscher AH, Fetzner UK, Bludau J et al (2011) Complications and management of complications in oesophageal surgery. Zentralbl Chir 136(3):213-223. https://doi.org/10.1055/s-00301262752

7. Lorenz D, Bruns CJ, Buhr HJ (2021) Statement of the surgical working group uppergastrointestinal tract (CAOGI) and the quality committee of the German Society for General and Visceral Surgery (DGAV) on the modified minimum caseload requirements for esophagus resection. Chirurg 92(11):1021-1024. https://doi.org/10.1007/s00104-021-01517-3

8. Hillner BE, Smith TJ (1998) Hospital volume and patient outcomes in major cancer surgery: a catalyst for quality assessment and concentration of cancer services. JAMA 280(20):1783-1784. https://doi.org/10.1001/jama.280.20.1783 


\section{Fachnachrichten}

9. Krautz C, Nimptsch U, Weber GF, Mansky T, Grützmann R (2018) Effect of hospital volume on in-hospital morbidity and mortality following pancreatic surgery in Germany. Ann Surg 267(3):411-417. https://doi.org/10.1097/SLA. 0000000000002248

10. Diers J, Baum P, Matthes H, Germer CT, Wiegering A (2021) Mortality and complication management after surgery for colorectal cancer depending on the DKG minimum amounts for hospital volume. Eur J Surg Oncol 47:850-857

11. Deijen CL, Tsai $A$, Koedam TWA, Veltcamp Helbach M, Sietses C, Lacy AM, Bonjer HJ, Tuynman JB (2016) Clinical outcomes and case volume effect of transanal total mesorectal excision for rectal cancer: a systematic review. Tech Coloproctol 20:811-824

12. Meyer HJ (2005) The influence of case load and the extent of resection on the quality of treatment outcome in gastric cancer. Eur J Surg Oncol 31(6):595-604. https://doi.org/10.1016/j. ejso.2005.03.002

13. Meyer H-J (2017) Mindestmengenregelungen erneut in der Diskussion DGCH und BDC zum aktuellen Krankenhaus-Report 2017. Pressekonferenz der Deutschen Gesellschaft für Chirurgie, 13. März 2017. https://www.dgch.de/index.php?id=99\&L=734\& tx_news_pi $1 \% 5 B$ news $\% 5 \mathrm{D}=837$ \&tx_news pi1\%5Bcontroller\%5D=News\&tx_news_pi1 $\% 5$ Baction $\% 5 \mathrm{D}=$ detail \&cHash $=44 \mathrm{e} 80 \mathrm{~d} 9 \mathrm{~b} 4 \mathrm{f} 2760$ 2ea58d3f5fd1d5e397.Zugegriffen: 18. Feb. 2022

14. Nimptsch U, Peschke D, Mansky T (2017) Mindestmengen und Krankenhaussterblichkeit - Beobachtungsstudie mit deutschlandweiten Krankenhausabrechnungsdaten von 2006 bis 2013. Gesundheitswesen 79(10):823-834. https://doi. org/10.1055/s-0042-100731. Epub 2016 Apr 6

15. Metzger R, Bollschweiler E, Hölscher AH et al (2004) High volume centers for esophagectomy: what is the number needed to achieve low postoperative mortality? Dis Esophagus 17:310-314

16. Fransen LFC, Berkelmans GHK, Asti E, Henegouwen MIVB, Berlth F, Bonavina L, Brown A, Bruns $C$, van Daele E, Gisbertz SS, Grimminger PP, Gutschow CA, Hannink G, Hölscher AH, Kauppi J, Lagarde SM, Mercer S, Moons J, Nafteux P, Nilsson M, Palazzo F, Pattyn P, Raptis DA Räsanen J, Rosato EL, Rouvelas I, Schmidt HM, Schneider PM, Schröder W, van der Sluis PC, Wijnhoven BPL, Nieuwenhuijzen GAP, Luyer MDP, EsoBenchmark Collaborative (2021) The effect of postoperative complications after minimally invasive esophagectomy on long-term survival:an international multicenter cohort study. Ann Surg 274(6):e1129-e1137. https://doi.org/10.1097/ SLA.0000000000003772

17. Hölscher AH, Fetzner UK (2021) Paraconduit hiatal hernia after esophagectomy. Preventionindication for surgery-surgical technique. Dis Esophagus 34(9):doab025. https://doi.org/10. 1093/dote/doab025

18. AOK-Bundesverband (2021) MindestmengenTransparenzliste 2022. Bundeweit. AOK. Die Gesundkeitskasse. Druckschrift. AOK-Bundesverband, Berlin

19. Kowalski C, Post S, Seufferlein T, Benz SR, Ferencz J, Wesselmann S (2018) Barriers and facilitating factors for research involvement in cancer centers: a survey of colorectal cancer center coordinators in Germany, Austria and Switzerland. Cancer Control 25(1):1073274818765475. https://doi. org/10.1177/1073274818765475

20. Osterloh F (2021) Krankenhausplan NRW Kooperation statt Konkurrenz. Dtsch Arztebl 118 B:1291

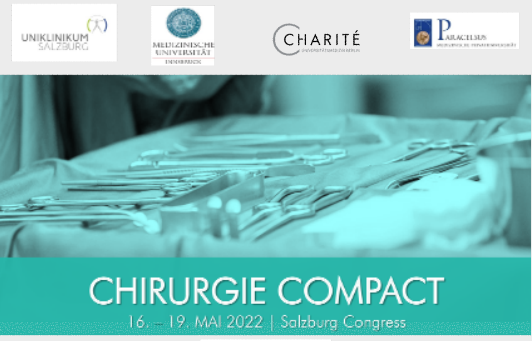

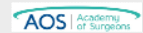

Chirurgie Compact 2022

Einladung nach Salzburg

Auf dem diesjährigen Fortbildungskongress Chirurgie Compact für junge und auch erfahrene Chirurginnen und Chirurgen werden international hochkarätige Referenten aktuelle Themen, praxisbezogene Tipps und Leitfäden für den Alltag präsentieren.

\section{Chirurgie Compact}

16.-19. Mai 2022 - Salzburg Congress Anmeldung und Infos finden Sie unter: https://www.academy-of-surgeons.com/chirurgie-compact/ 